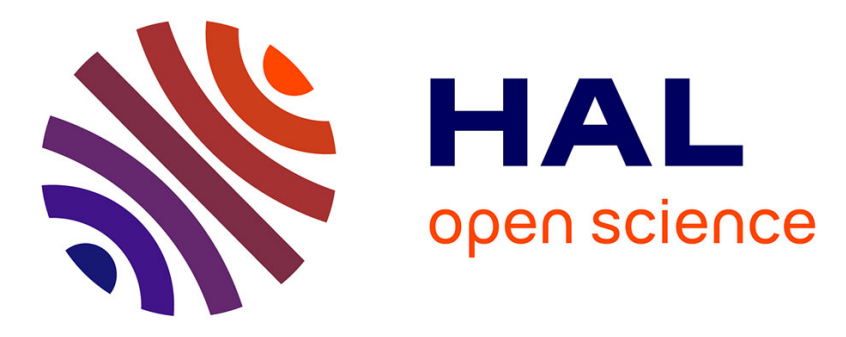

\title{
Sensor classification for the Fault Detection and Isolation problem
}

\author{
Christian Commault, Jean-Michel Dion, Sameh Yacoub Agha
}

\section{To cite this version:}

Christian Commault, Jean-Michel Dion, Sameh Yacoub Agha. Sensor classification for the Fault Detection and Isolation problem. SSSC 2007 - 3rd IFAC Symposium on System, Structure and Control, Oct 2007, Foz do Iguaçu, Brazil. hal-00149494

\section{HAL Id: hal-00149494 https://hal.science/hal-00149494}

Submitted on 25 May 2007

HAL is a multi-disciplinary open access archive for the deposit and dissemination of scientific research documents, whether they are published or not. The documents may come from teaching and research institutions in France or abroad, or from public or private research centers.
L'archive ouverte pluridisciplinaire HAL, est destinée au dépôt et à la diffusion de documents scientifiques de niveau recherche, publiés ou non, émanant des établissements d'enseignement et de recherche français ou étrangers, des laboratoires publics ou privés. 


\title{
SENSOR CLASSIFICATION FOR THE FAULT DETECTION AND ISOLATION PROBLEM
}

\author{
Christian Commault* Jean-Michel Dion* \\ Sameh Yacoub Agha*
* GIPSA-Lab, Department of Automatic Control, ENSIEG-INPG BP 46 38402 Saint Martin d'Hères, France.
Email: $\{$ Christian.Commault, Jean- Michel.Dion,Sameh.Yacoub-Agha\}@inpg.fr

\begin{abstract}
In this paper we deal with the observer based Fault Detection and Isolation problem. When this FDI problem is solvable with the existing sensors, we will classify these sensors according to their importance for the solvability of the considered FDI problem. The sensors will be classified into essential sensors, useless sensors and useful sensors. We tackle this problem when the system under consideration is structured, that is, the entries of the system matrices are either fixed zeros or free parameters. With such structured systems one can associate a graph. Simple algorithms allow to get from the graph the above sensor classification.
\end{abstract}

Keywords: Linear systems, Structured systems, Fault detection and isolation, Sensor classification.

\section{INTRODUCTION}

The Fault Detection and Isolation (FDI) problem has received considerable attention in the past ten years (Chen and Patton, 1999; Frank, 1996). It consists of building residuals from the available data and isolating, whenever possible, the faults using the residuals. When this FDI problem is solvable with the existing sensors, we will classify these sensors according to their importance in the solvability of the considered FDI problem. The sensors will be classified into essential sensors, useless sensors and useful sensors. In simple words the failure of an essential sensor will lead to the non solvability of the FDI problem while the failure of a useless sensor will not affect the solvability of the FDI problem. The useful sensors are simply those which are not useless.

We consider the FDI problem in the framework of structured linear systems which represent a large class of parameter dependent systems (Lin, 1974; Dion et al., 2003), that is the entries of the system matrices are either fixed zeros or free parameters. With such structured systems one can associate a graph and simple properties of these graphs will allow us to get interesting structural information on the system. The FDI problem has been tackled in this context, see (Commault et al., 2002; Verde, 2005). When the FDI problem has no solution with the existing sensors a structural analysis has allowed to determine the number and the location of needed additional sensors (Commault and Dion, 2007; Commault et al., 2006b).

Using the graph associated with the system, we will prove that useless sensors correspond to output vertices which are not connected to faults by a path. One can determine in the system associated 
graph a set of bottleneck vertices called "essential vertices", the output vertices in this set indeed correspond to the essential sensors. The sensor classification can be performed using simple polynomial complexity algorithms. In (Commault et al., 2006a) a similar sensor classification has been proposed for the case of generic observability. The paper is structured as follows. The problem is formulated in Section 2. Structured systems are presented in Section 3. In Section 4, we provide with a characterization of useless and essential sensors for the observer-based FDI problem. Numerical aspects are considered in Section 5. Concluding remarks end the paper.

\section{PROBLEM FORMULATION}

\subsection{Observer-based FDI problem}

Let us consider the following linear time-invariant system :

$$
\Sigma\left\{\begin{array}{c}
\dot{x}(t)=A x(t)+L f(t)+B u(t) \\
y(t)=C x(t)+M f(t)
\end{array} .\right.
$$

where $x(t) \in \mathbb{R}^{n}$ is the state vector, $f(t) \in \mathbb{R}^{r}$ the fault vector, $u(t) \in \mathbb{R}^{m}$ is the control input vector and $y(t) \in \mathbb{R}^{p}$ the measured output vector. Each output variable is provided by a specific sensor. For the sake of simplicity, in the following we will denote by $y_{i}$ either the $i$ th sensor or the $i$ th output variable. $A, C, B, L$ and $M$ are matrices of appropriate dimensions.

A dedicated residual set is designed using a bank of $r$ observers for system (1), according to the dedicated observer scheme (Chen and Patton, 1999).

The $i$ th observer of this bank of $r$ observers is designed for a system of type (1) as follows:

$$
\dot{\hat{x}}^{i}(t)=A \hat{x}^{i}(t)+K^{i}\left(y(t)-C \hat{x}^{i}(t)\right)+B u(t),
$$

where $\hat{x}^{i}(t) \in \mathbb{R}^{n}$ is the state of the $i$ th observer, $K^{i}$ is the observer gain to be designed such that $\hat{x}^{i}(t)$ asymptotically converges to $x(t)$, when $f(t)=0$.

The residuals are defined as :

$$
r_{i}(t)=Q^{i}\left(y(t)-C \hat{x}^{i}(t)\right), \text { for } i=1, \ldots, r,
$$

where $Q^{i}$ is a $1 \times p$ matrix.

Definition 1. Let $\Sigma$ be a linear system as in Equation (1) with the set of sensors $Y=\left\{y_{1}, \ldots, y_{p}\right\}$. The bank of observer-based FDI problem associated with $Y$ consists in finding, if possible, matrices $K^{i}$ and $Q^{i}$, such that, for $i=1,2, \ldots, r, A-$ $K^{i} C$ is stable, and the fault to residual transfer matrix is non zero, proper and diagonal.
It is clear that the solvability of this problem relies on the available sensors, a sufficient amount of information is needed to detect and isolate the faults via the dedicated observer scheme.

\subsection{Sensor classification for FDI}

In this section we provide with a classification of the system sensors with respect to their importance concerning the bank of observer based FDI problem of Definition 1. In this study we suppose that the FDI problem has a solution with the given sensors. The sensors will be classified into essential sensors, useless sensors and useful sensors. In simple words the failure of an essential sensor will lead to the non solvability of the FDI problem while the failure of an useless sensor will not affect the solvability of the FDI problem.

Let us first define what we mean by a solution for the FDI problem with a given subset of sensors.

Definition 2. Let $\Sigma$ be the linear system of Equation (1) and assume that the FDI problem of Definition 1 has a solution with the sensor set $Y$. Let $V$ be a subset of $Y$. $V$ is a solution of the bank of observer based FDI problem of Definition 1 , if the considered FDI problem remains solvable with the subset of sensors $V$.

Define now the essential and useless sensors:

Definition 3. Consider the linear system $\Sigma$ of Equation (1). Let $y_{i}$ be the $i$ th sensor of the system.

The sensor $y_{i}$ is essential if $y_{i}$ belongs to any solution of the FDI problem in the sense of Definition 2 .

Definition 4. Consider the linear system $\Sigma$ of Equation (1). Let $y_{i}$ be the $i$ th sensor of the system and $V /\left\{y_{i}\right\}$ the set $V$ from which the sensor $y_{i}$ has been removed.

The sensor $y_{i}$ is useless if for any solution $V$ containing $y_{i}$ of the FDI problem in the sense of Definition $2, V /\left\{y_{i}\right\}$ is still a solution.

\section{LINEAR STRUCTURED SYSTEMS}

\subsection{Definitions and basic properties}

We will consider models based on the available physical knowledge on the system. These models capture the relations between internal variables but without fixing the precise value of the parameters. Frequently, starting from a nonlinear dynamical model of a system one gets linearized models around different set points which share the 
same structure but with different parameter values. A distillation column example is worked out in (Commault and Dion, 2006). This is illustrated in Figure 1 where a nonlinear model is linearized around different set points.

Such models which incorporate prior knowledge

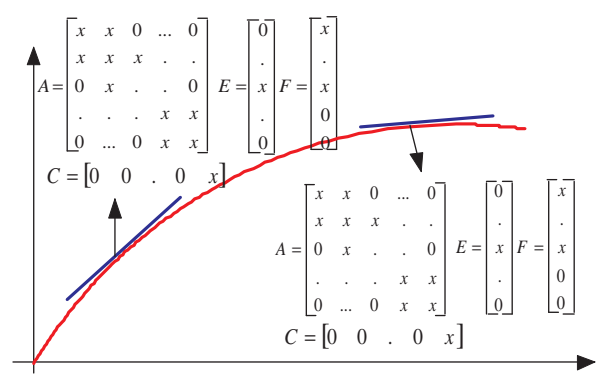

Fig. 1. Nonlinear system

on the structure have been often used in the literature see e.g (Lin, 1974; Blanke et al., 2003)

In this paper we will consider linear structured systems as in (Lin, 1974). We consider linear systems as described in (1), but with parameterized entries and denoted by $\Sigma_{\Lambda}$

$$
\Sigma_{\Lambda}\left\{\begin{array}{c}
\dot{x}(t)=A x(t)+L f(t)+B u(t) \\
y(t)=C x(t)+M f(t)
\end{array} .\right.
$$

This system is called a linear structured system if the entries of the composite matrix $J=$ $\left[\begin{array}{ccc}A & L & B \\ C & M & 0\end{array}\right]$ are either fixed zeros or independent parameters (not related by algebraic equations). $\Lambda=\left\{\lambda_{1}, \lambda_{2}, \ldots, \lambda_{k}\right\}$ denotes the set of independent parameters of the composite matrix $J$. More details can be found in (Dion et al., 2003).

For such systems one can study generic properties i.e. properties which are true for almost all values of the parameters collected in $\Lambda$ (Murota, 1987). A directed graph $G\left(\Sigma_{\Lambda}\right)=(V, W)$ can be easily associated with the structured system $\Sigma_{\Lambda}$ of type (4) where the matrix $\left[\begin{array}{ccc}A & L & B \\ C & M & 0\end{array}\right]$ is structured:

- the vertex set is $V=U \cup F \cup X \cup Y$ where $U, F, X$ and $Y$ are the control input, fault, state and output sets given by $\left\{u_{1}, u_{2}, \ldots, u_{m}\right\}$, $\left\{f_{1}, f_{2}, \ldots, f_{r}\right\},\left\{x_{1}, x_{2}, \ldots, x_{n}\right\}$ and $\left\{y_{1}, y_{2}, \ldots\right.$, $\left.y_{p}\right\}$ respectively,

- the arc set is $W=\left\{\left(u_{i}, x_{j}\right) \mid B_{j i} \neq 0\right\} \cup$ $\left\{\left(f_{i}, x_{j}\right) \mid L_{j i} \neq 0\right\} \cup\left\{\left(x_{i}, x_{j}\right) \mid A_{j i} \neq 0\right\} \cup\left\{\left(x_{i}, y_{j}\right) \mid C_{j i}\right.$ $\neq 0\} \cup\left\{\left(f_{i}, y_{j}\right) \mid M_{j i} \neq 0\right\}$, where $A_{j i}$ (resp. $B_{j i}$ $\left.C_{j i}, L_{j i}, M_{j i}\right)$ denotes the entry $(j, i)$ of the matrix $A$ (resp. $B, C, L, M$ ).

Recall that a directed path in $G\left(\Sigma_{\Lambda}\right)$ from a vertex $i_{0}$ to a vertex $i_{q}$ is a sequence of arcs $\left(i_{0}, i_{1}\right),\left(i_{1}, i_{2}\right), \ldots,\left(i_{q-1}, i_{q}\right)$ such that $i_{t} \in V$ for $t=0,1, \ldots, q$ and $\left(i_{t-1}, i_{t}\right) \in W$ for $t=$ $1,2, \ldots, q$. If $i_{0} \in F$ and, $i_{q} \in Y, P$ is called a fault-output path. If $i_{0} \in V_{1}$ and, $i_{l} \in V_{2}$, where $V_{1}$ and $V_{2}$ are two subsets of $V, P$ is called a $V_{1}-V_{2}$ path. Moreover, if $i_{0}$ is the only vertex of $P$ which belongs to $V_{1}$ and $i_{l}$ is the only vertex of $P$ which belongs to $V_{2}, P$ is called a direct $V_{1}-V_{2}$ path.

A set of paths with no common vertex is said to be vertex disjoint. A $V_{1}-V_{2}$ linking of size $k$ is a set of $k$ vertex disjoint $V_{1}-V_{2}$ paths. A linking is maximal when $k$ is maximal.

In this paper we will consider that $\Sigma_{\Lambda}$ is structurally observable (Lin, 1974; Murota, 1987). This implies that any state vertex is linked to output (sensor) vertices by a path in $G\left(\Sigma_{\Lambda}\right)$.

Give now the result concerning the diagonal FDI problem by using a bank of observers, which was stated first in (Commault et al., 2002).

Theorem 5. Consider the structurally observable system with $r$ faults $\Sigma_{\Lambda}$ as defined in (4) and the associated graph $G\left(\Sigma_{\Lambda}\right)$. The bank of observerbased diagonal FDI problem of Definition 1, is generically solvable if and only if:

$$
k=r,
$$

where $k$ is the size of a maximal fault-output linking in $G\left(\Sigma_{\Lambda}\right)$.

\subsection{The essential vertices and the minimal output separator}

Most of the basic material of this subsection is based on (van der Woude, 2000). A separator $S$ of $G\left(\Sigma_{\Lambda}\right)$ is a set of vertices such that any fault-output path has at least one vertex in $S$. Separators with a minimal number of vertices are called minimal. A classical result is that the minimal size of a separator is the maximal size of a fault-output linking.

Definition 6. The set of essential vertices $V_{\text {ess }}$ of $G\left(\Sigma_{\Lambda}\right)$ is the set of vertices which belong to any maximal size fault-output linking.

Definition 7. The minimal output separator of $G\left(\Sigma_{\Lambda}\right), S_{*}$, is the set of begin vertices of all direct $V_{e s s}-Y$ paths.

It can be shown that $S_{*}$ is a separator of minimal dimension. $S_{*}$ is indeed the last bottleneck between faults and outputs. $S_{*}$ may contain fault, state and output vertices.

\section{Example 1}

To illustrate the previous notions, let us deal with a first example with no control input, 6 faults and 8 sensors, which graph $G\left(\Sigma_{\Lambda}\right)$ is given in Figure 2. One can show that the corresponding set of essential vertices is $V_{\text {ess }}=$ $\left\{f_{1}, \ldots, f_{6}, x_{3}, x_{4}, x_{5}, x_{6}, y_{1}, y_{2}, y_{3}, y_{5}\right\}$, see Figure 3 . The corresponding minimal output separator is $S_{*}=\left\{x_{4}, x_{6}, y_{1}, y_{2}, y_{3}, y_{5}\right\}$, see Figure 4 . 


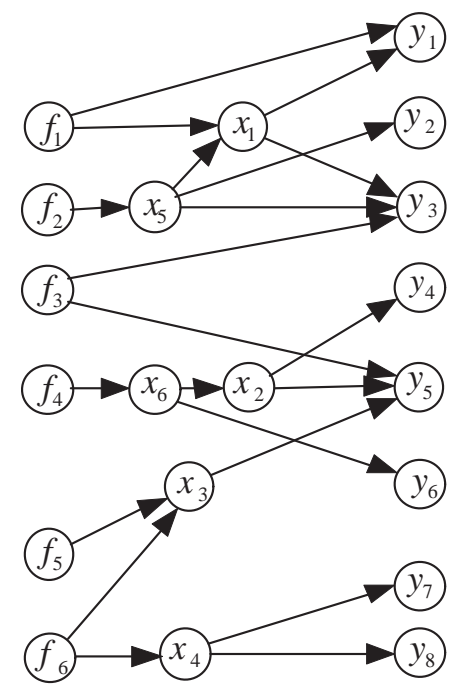

Fig. 2. Graph $G\left(\Sigma_{\Lambda}\right)$ of Example 1.

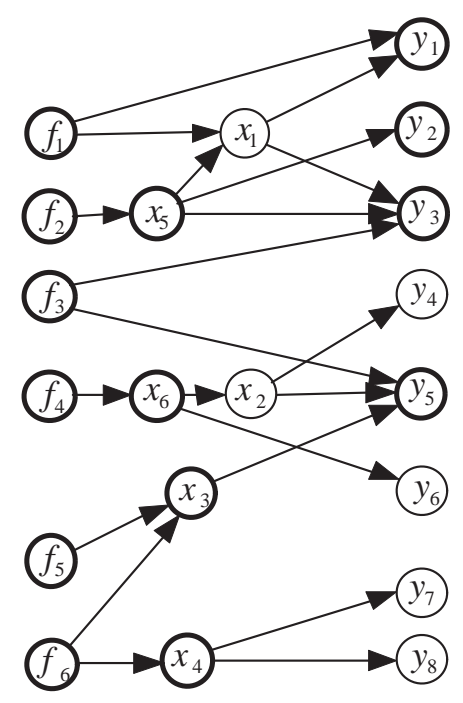

Fig. 3. Essential vertices of Example 1.

\section{SENSOR CHARACTERIZATION}

By using Theorem 5, one can easily characterize solutions of the FDI problem of Definition 2 in the following way.

Proposition 8. Consider the structurally observable system $\Sigma_{\Lambda}$ with $r$ faults and sensor set $Y$ as defined in (4) and the associated graph $G\left(\Sigma_{\Lambda}\right)$. Let $V$ be a subset of $Y$. $V$ is a solution of the generic bank of observer based diagonal FDI problem if and only if there exists an $F-V$ linking of size $r$ in $G\left(\Sigma_{\Lambda}\right)$.

The useless sensors of Definition 4 for the FDI can then be characterized as follows.

Theorem 9. Consider the linear structured system $\Sigma_{\Lambda}$ with sensor set $Y$ and its associated graph $G\left(\Sigma_{\Lambda}\right)$. A sensor $y_{i} \in Y$ is useless if and only if

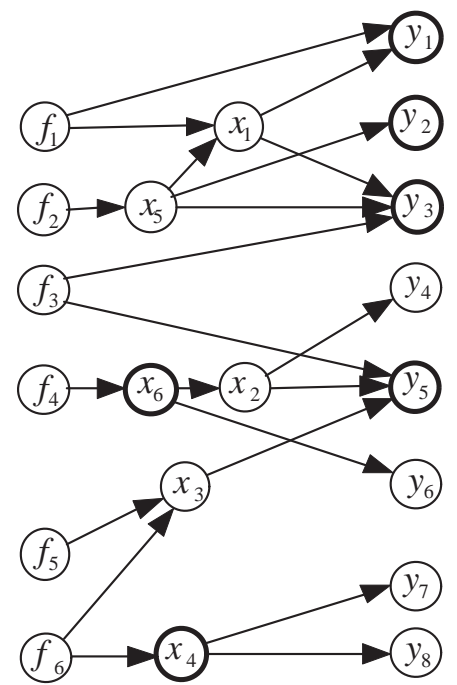

Fig. 4. Output separator of Example 1.

there is no $F-\left\{y_{i}\right\}$ path in $G\left(\Sigma_{\Lambda}\right)$, where $F$ is the fault set.

\section{Proof}

Sufficiency Assume that there is no $F-\left\{y_{i}\right\}$ path in $G\left(\Sigma_{\Lambda}\right)$ and let $V$ be a solution such that $y_{i} \in V$. $V$ being a solution, from Proposition 8 there is an $F$ - $V$ linking of size $r$ in $G\left(\Sigma_{\Lambda}\right)$. This linking is not incident to $y_{i}$ since there is no $F-\left\{y_{i}\right\}$ path. Therefore this linking is also a size $r$ linking from $F$ to $V /\left\{y_{i}\right\}$ then $V /\left\{y_{i}\right\}$ is a solution which proves that $y_{i}$ is useless.

Necessity Assume now that there is an $F-\left\{y_{i}\right\}$

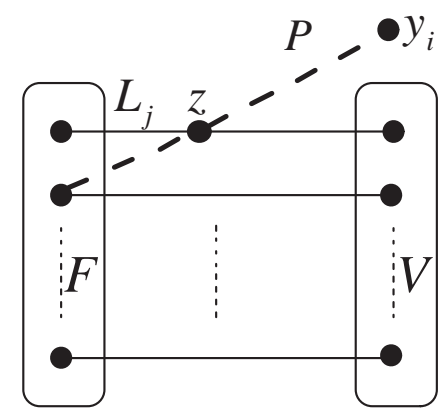

Fig. 5. The linking $\mathcal{L}$ and the path $P$.

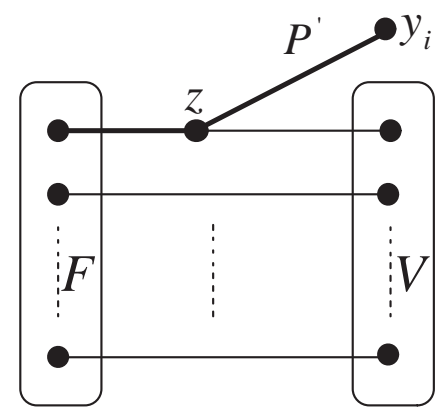

Fig. 6. The new linking $\mathcal{L}^{\prime}$. 
path $P$ in $G\left(\Sigma_{\Lambda}\right)$ and consider an $F-Y$ linking $\mathcal{L}$ of size $r$ in $G\left(\Sigma_{\Lambda}\right)$, which is then maximal. Denote by $V$ the subset of $Y$ consisting of all the outputs incident to $\mathcal{L}$. $V$ is clearly a solution for the FDI problem. Assume first that $y_{i} \in V$, then $V /\left\{y_{i}\right\}$ is not a solution because it has cardinality $(r-1)$. Therefore $y_{i}$ is not useless. Suppose now that $y_{i}$ does not belong to $V$. A given $F-\left\{y_{i}\right\}$ path $P$ must have common vertices with the linking $\mathcal{L}$, otherwise $\mathcal{L}$ would not be maximal. Concentrate now on the $F-Y$ path $L_{j}$ of $\mathcal{L}$ which contains the last common vertex $z$ between $\mathcal{L}$ and $P$ when travelling along $P$ from $F$ to $y_{i}$, see Figure 5. By concatenating the part of $L_{j}$ from $F$ to $z$ and the part of $P$ from $z$ to $y_{i}$ we get a new path $P^{\prime}$ which is incident to $y_{i}$ and having no common vertex with $\mathcal{L} / L_{j}$. We get a new linking $\mathcal{L}^{\prime}=P^{\prime} \cup\left(\mathcal{L} / L_{j}\right)$ of size $r$ which is incident to $y_{i}$, see Figure 6 . Therefore $y_{i}$ belongs to a solution $V^{\prime}$ such that $V^{\prime} /\left\{y_{i}\right\}$ is not a solution, then $y_{i}$ is not useless.

Theorem 10. Consider the linear structured system $\Sigma_{\Lambda}$ with its associated graph $G\left(\Sigma_{\Lambda}\right)$. The set of essential sensors is given by $Y_{e}=Y \cap V_{e s s}$, where $Y$ is the sensor set and $V_{\text {ess }}$ is the set of essential vertices of $G\left(\Sigma_{\Lambda}\right)$.

\section{Proof}

Sufficiency Assume that $y_{i} \in Y_{e}=Y \cap V_{\text {ess }}$. Then any $F-Y$ linking of size $r$ is incident to $y_{i}$ and any linking $F-\left\{Y / y_{i}\right\}$ has size less than or equal to $r-1$. This is a fortiori true for for any $F-\left\{V / y_{i}\right\}$ linking with $V \subseteq Y$ therefore $y_{i}$ must belong to any solution and $y_{i}$ is essential.

Necessity If $y_{i} \notin Y_{e}=Y \cap V_{\text {ess }}$, then $y_{i}$ does not belong to any maximal matching. Therefore there exists a size $r$ linking which is not incident to $y_{i}$. The set $V$ of end vertices of the paths of this linking is a solution of the FDI problem which does not contain $y_{i}$, then $y_{i}$ is not essential .

\section{Example 2}

Consider the linear structured system with 3 control inputs, 6 faults and 10 sensors illustrated in Figure 7 . The corresponding set of essential vertices is $V_{e s s}=\left\{y_{1}, y_{2}, f_{1}, \ldots, f_{6}, x_{1}, \ldots, x_{6}, x_{9}, \ldots\right.$, $\left.x_{14}\right\}$, see Figure 8 .

- The sensors $y_{1}$ and $y_{2}$ are essential sensors because they belong to $Y \cap V_{\text {ess }}$.

- The sensors $y_{9}$ and $y_{10}$ are useless sensors because they belong to no $F-Y$ path.

- The sensors $y_{1}, \ldots, y_{8}$, are useful since they are not useless.

\section{COMPUTATIONAL ASPECTS}

In this section we give some indications on the way for computing the sets of useless and essential sensors. Theorem 10 which characterizes the

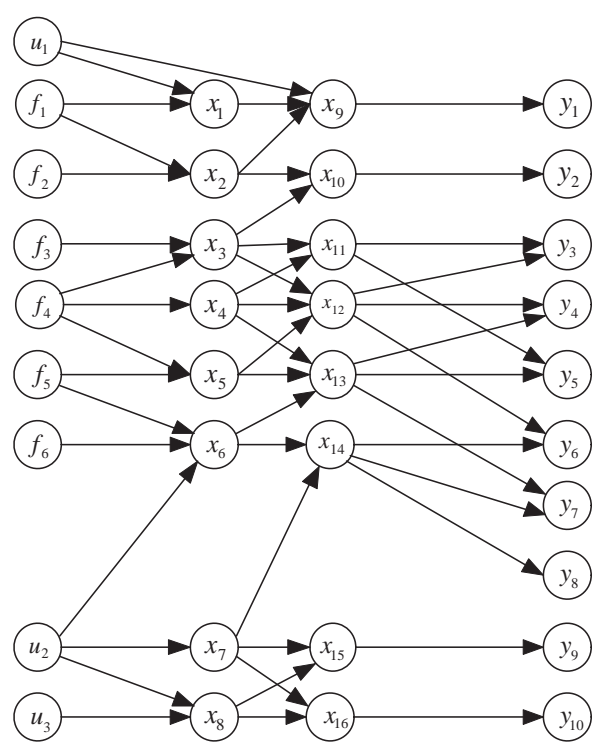

Fig. 7. The graph $G\left(\Sigma_{\Lambda}\right)$ of Example 2.

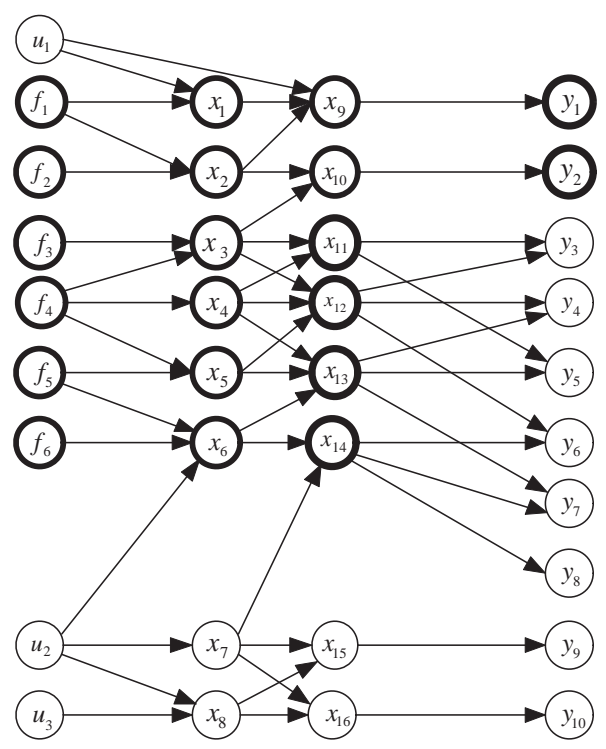

Fig. 8. Essential vertices of Example 2.

essential sensors can be reformulated as follows using the concept of minimal output separator.

Proposition 11. Consider the linear structured system $\Sigma_{\Lambda}$ with its associated graph $G\left(\Sigma_{\Lambda}\right)$. The set of essential sensors is given by $Y_{e}=Y \cap S_{*}$, where $Y$ is the sensor set and $S_{*}$ is the minimal output separator of $G\left(\Sigma_{\Lambda}\right)$.

It turns out that the set $S_{*}$ can be computed using max flow techniques as the Ford and Fulkerson algorithm (Murota, 1987). This kind of algorithm is of polynomial complexity allowing to deal with large scale systems.

The set of useless sensors can be obtained by using a marking procedure starting from the fault vertices and walking along $F-Y$ paths. The output vertices which will not be marked will 
correspond to useless sensors. This very simple algorithm is also polynomial.

\section{CONCLUDING REMARKS}

In this paper we have classified the sensors with respect to their importance for the solvability of the observer based FDI problem. The proposed analysis is mainly based on the system structure and is parameter independent. This analysis reveals the critical sensors which should be comforted in order to perform a robust FDI. The number of useful but non essential sensors give a measurement of their degree of redundancy concerning the FDI problem.

\section{REFERENCES}

Blanke,

M.,

M. Kinnaert, J. Lunze and M. Staroswiecki (2003). Diagnosis and fault-tolerant control. Springer-Verlag.

Chen, J. and R.J. Patton (1999). Robust modelbased fault diagnosis for dynamic systems. Kluwer Academic Publishers.

Commault, C. and J.M. Dion (2006). Approche structurelle du diagnostic, application à un modèle de colonne à distiller. CIFA Bordeaux.

Commault, C. and J.M. Dion (2007). Sensor location for diagnosis in linear systems: a structural analysis. IEEE Transactions on Automatic Control 52(2), 155-169.

Commault, C., J.M. Dion and D.H. Trinh (2006a). Observability preservation under sensor failure. $C D C$ San Diego.

Commault, C., J.M. Dion and S. Yacoub Agha (2006b). Structural analysis for sensor location in fault detection and isolation. IFAC SafeProcess. Beijing.

Commault, C., J.M. Dion, O. Sename and R. Motyeian (2002). Observer-based fault detection and isolation for structured systems. IEEE Trans. Automat. Control 47(12), 2074-2079.

Dion, J.M., C. Commault and J. van der Woude (2003). Generic properties and control of linear structured systems: a survey. Automatica 39(7), 1125-1144.

Frank, P.M. (1996). Analytical and qualitative model-based fault diagnosis - a survey and some new results. European Journal of Control 2, 6-28.

Lin, C.T. (1974). Structural controllability. IEEE Trans. Automat. Control 19, 201-208.

Murota, K. (1987). Systems Analysis by Graphs and Matroids. Vol. 3 of Algorithms and Combinatorics. Springer-Verlag New-York, Inc.

van der Woude, J.W. (2000). The generic number of invariant zeros of a structured linear system. SIAM Journal. of Control 38, 1-21.
Verde, C. (2005). Faults isolation for nonlinear Hessenberg systems. American Control Conference, Portland. 